\title{
Basilar artery thrombosis- a ticking time bomb
}

\begin{abstract}
Basilar artery occlusion (BAO) accounts for $6-10 \%^{1}$ of strokes, causing significant morbidity and mortality often with a poor prognosis..$^{1-3}$ Conventional stroke treatment with intravenous thrombolysis (IVT) is thought to be less effective in large vessel occlusion such as BAO due to a higher clot burden. ${ }^{4}$ In this paper we report the case of a patient who only presented with symptoms in the chronic rather than acute phase of BAO and review the literature regarding mechanical management of this condition.Emerging evidence suggests mechanical intervention in chronic $\mathrm{BAO}$ is possible and may still achieve good outcomes providing an option in patients like ours who fail to respond to conventional thrombolysis and secondary preventative medical management.
\end{abstract}

Keywords: Basilar artery, Occlusion, Stroke, Mechanical, Chronic
Volume 2 Issue 2 - 2015

\begin{abstract}
Katherine Metcalfe, Ganesh Subramanian
Department of Stroke Medicine, Nottingham University Hospitals NHS Trust, UK
\end{abstract}

\begin{abstract}
Correspondence: Katherine Metcalfe, Department of Stroke Medicine, Nottingham University Hospitals NHS Trust, Hucknall Road, Nottingham, NG5 IPB, UK, Tel 773I754340, Email katherinemetcalfe@nhs.net
\end{abstract}

Received:September 22, 2015 | Published: April 08, 2015
Abbreviations: BAO, Basilar Artery Occlusion; IVT, Intravenous Thrombolysis; ECG, Electrocardiogram; CT, Computed Tomography; NIHSS, National Institute of Health Stroke Scale; INR, Interventional Neuroradiologist; DWI, Diffusion-Weighted Imaging; pc-ASPECTS, Posterior circulation-Acute Stroke Prognosis Early CT score; CTASI, CT Angiography Source Images

\section{Introduction}

Basilar artery occlusion (BAO) accounts for $6-10 \%$ of strokes and causes significant morbidity and mortality. ${ }^{1-3,5}$ Treatment is challenging due to a limited evidence base. We discuss a patient presenting with $\mathrm{BAO}$, highlighting new developments surrounding intervention in chronic BAO.

\section{Case presentation}

A 61 year old gentleman, previously independent 50 pack year smoker, with a background of hyperlipidaemia, ischaemic heart disease and peripheral vascular disease presented with sudden onset of left-sided numbness and dysarthria. On examination, he was hypertensive with a left-sided facial droop, left pronator drift and mild dysarthria. His ECG showed sinus rhythm. CT brain scan showed small cerebellar infarcts and hyperdensity of the basilar artery with $\mathrm{CT}$ angiogram revealing distal BAO (Figure 1). He was thrombolysed with intravenous alteplase despite an NIHSS of 4 as per local protocol for BAO. Interventional neuroradiologists (INR) felt the risks of mechanical thrombectomy outweighed the benefits as he had presented with mild basilar syndrome. Despite initial improvement and appropriate secondary prevention, he developed oculomotor palsies, drowsiness and ataxia. MR imaging revealed new posterior circulation infarcts (Figure 2) with persisting BAO. On rediscussion, the INR felt the clot was chronic and as mechanical intervention in chronic BAO is evidence-sparse, he was medically managed with dual antiplatelets, statin, antihypertensives and rehabilitation. On discharge, his modified Rankin score was 3.

\section{Discussion}

$\mathrm{BAO}$ is relatively rare, accounting for $6-10 \%$ of strokes. Although women tend to be affected later in life than men, most patients who develop BAO are aged 50-80. Predominant risk factors include hypertension, diabetes, ischaemic heart disease, smoking, hyperlipidaemia, previous stroke and peripheral vascular disease. The most common underlying aetiology for BAO is atherosclerosis. However embolic phenomena, vasculitis, dissection and even migraine may also be implicated. Classically, patients experience transient ischaemic attacks or a prodrome of symptoms including headache and vertigo in the weeks preceding the acute stroke. ${ }^{1}$ However this may not always be present, as was the case with our patient who developed abnormal neurology likely secondary to embolic phenomenon from his chronic BAO.

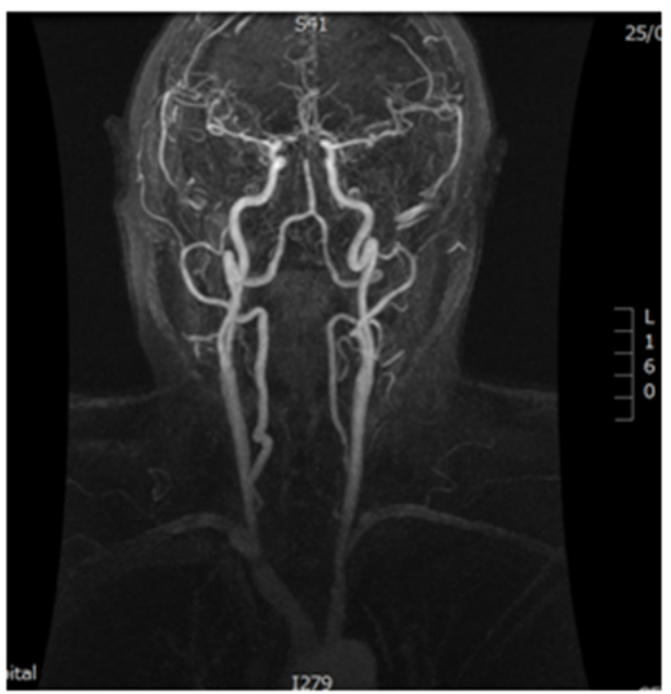

Figure I MRA showing occlusion of the distal basilar artery pre-thrombolysis.

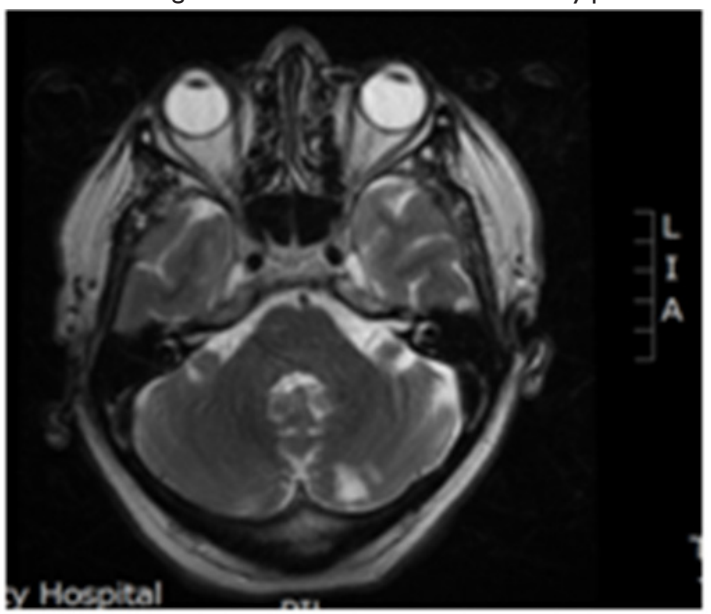

Figure 2 Follow-up MRI post-thrombolysis, showing multiple posterior circulation infarcts. 
Prognosis is extremely variablewith poor outcome (defined as death, dependency, severe disability or modified Rankin score 3-6) in 54-95\% of cases but factors thought to be associated with worse prognosis include embolic aetiology, distal artery involvement, occlusion rather than stenosis and presentation with decreased consciousness, tetraparesis, abnormal pupils or bulbar signs. Achievement of recanalisation is thought to be the biggest factor influencing clinical outcome with even partial recanalisation providing some benefit. ${ }^{1,4}$

Whilst most patients present in the acute phaseof BAO, some patients, such as ours, present in the chronic phase or survive the acute phase but have recurrent ischaemic events due to suboptimal recanalisation; however, evidence-based guidance for management of chronic basilar artery occlusion is sparse. Robust evidence supports the use of IVT in acute strokes, ${ }^{6}$ but there is little, if any, evidence regarding the use of both IVT and intra-arterial thrombolysis (IAT) as stand-alone therapies in chronic occlusion. Some evidence suggests that IVT is also less effective in strokes caused by large vessel occlusion such as $\mathrm{BAO}^{4}$ due to high clot burden, as it was in our patient. Although early recanalisation is likely to result in better functional outcomes in $\mathrm{BAO},{ }^{7}$ it is also thought that the brainstem tolerates ischaemia better than the cerebral hemispheres ${ }^{2,8}$ suggesting treatment benefit is still possible after recanalisation of a chronic occlusion $^{2,8}$ even if initial therapy with IVT in the acute phase fails. In this instance, other therapies such as mechanical recanalisation may be considered, as they may achieve superior revascularisation with more immediate effect than thrombolysis. ${ }^{4}$ Most reports allude to the use of endovascular techniques, particularly mechanical clot extractionfor treatment of chronic $\mathrm{BAO}^{2,3,9,10}$ with thrombolysis alone having only been used up to 79 hours post symptom onset. ${ }^{2}$

Case reports have described mechanical intervention in chronic BAO ranging from 4 to 80 days post stroke in 16 patients. ${ }^{2,3,9,10}$ Balloon angioplasty followed by stent deployment was used for 14 of these patients to achieve recanalization..$^{3,9,10}$ In the other 2 instances, mechanical thrombectomy followed by balloon angioplasty was used. ${ }^{2}$ In some cases localized intra-arterial thrombolysis was used to aid mechanical management by allowing the instruments to traverse the clot. ${ }^{3,5}$ Recanalisation was achieved in 15 out of 16 patients. Of these 16 patients, 6 suffered periprocedural complications ${ }^{5,9}$ and $2 \operatorname{died}^{9}$ from the complications. Reported complications included subarachnoid haemorrhage and arterial dissection. ${ }^{5,9}$ Some authors felt there was a higher risk of complication in chronic BAO. ${ }^{3,5,9}$ All patients who survived the procedure were discharged with antiplatelet therapy. $2,3,9,10$

Radiographic follow up varied between authors from 3-8 months. Three patients developed significant in-stent stenosis $^{3,9}$ which was asymptomatic in one case. ${ }^{3}$ Clinical follow up varied from 3-30 months. Whilst there was no consistent method used by all authors, significant improvement in symptoms and functioning was reported for 10 patients, 1 patient remained stable, 1 was unable to be recanalized and 4 died ( 2 from complications of the procedure). These reports suggest good functional outcome is still possible, even in patients that had presented with severe symptoms, and there may be benefit to intervention in chronic BAO. These are only anecdotes and there is no randomized controlled trial evidence to guide the treating physician when they come across this rare presentation.

Determining patients with potential to benefit from treatment of chronic BAO is controversial. Whilst severe symptoms have been associated with poor prognosis, some of these patients achieved good functional outcomes with intervention, ${ }^{2}$ suggesting symptom severity should not preclude treatment. Equally intervention has improved functional outcome in patients up to 80 days post-symptom onset implying exclusion by time criteria may also be unreasonable. The use of radiological scores may be more reliable for predicting benefit potential with the brainstem DWI score, pc-ASPECTS and CTASI scores proposed as possible indicators but further research is

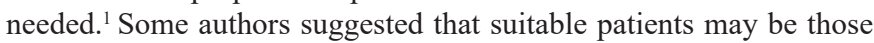
who experience recurrent ischaemic events and disabling symptoms despite best medical treatment as was the case with our patient. ${ }^{5,9}$

In conclusion, BAO is challenging to treat and may not respond to conventional IVT. Mechanical intervention could be considered for patients who present with chronic BAO or in whom treatment of the acute phase fails to result in recanalisation and resolution of symptoms but it is yet unclear what criterion could be used in selecting appropriate patients. It is difficult to undertake a randomized controlled trial in such patients due to the difficulty in diagnosing and the infrequent occurrence of chronic BAO (let alone the ethical dilemma the treating physicians may have).

\section{Acknowledgments}

None.

\section{Conflicts of interest}

None.

\section{References}

1. Mortimer AM, Bradley M, Renowden SA. Endovascular therapy for acute basilar artery occlusion: a review of the literature. $J$ Neurointerv Surg. 2012;4(4):266-273.

2. Noufal M, Schmidley JW, Erdem E, et al. Basilar artery occlusion treated with mechanical thrombectomy beyond eight hours with successful recanalisation and good functional outcomes. Cerebrovasc Dis. 2009;27(6):614-615.

3. Yu W, Kostanian V, Fisher M. Endovascular recanalization of basilar artery occlusion 80 days after symptom onset. Stroke. 2007;38(4):13871389.

4. Smith WS, Sung G, Saver J, et al. Mechanical thrombectomy for acute ischaemic stroke - final results of the multi MERCI trial. Stroke. 200839(4):1205-1212.

5. Lin R, Aleu A, Jankowitz B, et al. Endovascular revascularisation of chronic symptomatic vertebrobasilar occlusion. $J$ Neuroimaging. 201222(1):74-79.

6. IST-3 collaborative group, Sandercock P, Wardlaw JM, et al. The benefits and harms of intravenous thrombolysis with recombinant tissue plasminogen activator within $6 \mathrm{~h}$ of acute ischaemic stroke (the third international stroke trial [IST-3]): a randomised controlled trial. Lancet. 2012379(9834):2352-2363.

7. Vergouwen MD, Algra A, Pfefferkorn T, et al. Time is brain(stem) in basilar artery occlusion. Stroke. 2012;43(11):3003-3006.

8. Axer H, Grassel D, Bramer D, et al. Time course of diffusion imaging in acute brainstem infarcts. J Magn Reson Imaging. 2007;26(4):905-912.

9. Dashti SR, Park MS, Stiefel MF, et al. Endovascular recanalization of the subacute to chronically occluded basilar artery: initial experience and technical considerations. Neurosurgery. 2010;66(4):825-832.

10. Liu XC, Chen C, Shi MC, et al. Revascularisation of vertebrobasilar artery occlusion at the chronic stage. Neurosciences (Riyadh). 2013;18(3):269-272. 